\title{
A Novel Algorithm for Minutiae Matching
}

\author{
Om Preeti Chaurasia \\ Amity School of Engineering and Technology, Amity University \\ Noida, India \\ E-mail: preeti.princy.chaurasia@gmail.com \\ Saumya Ranjan Giri \\ IL\&FS Technologies Ltd. \\ Bhubaneswar, India \\ E-mail: saumya.giri07@gmail.com \\ Anchal Garg \\ Amity School of Engineering and Technology, Amity University \\ Noida, India \\ E-mail: agarg@amity.edu
}

\begin{abstract}
This paper presents a simple and novel algorithm for minutiae matching in fingerprint images. After correct detection of all minutiae in two fingerprint images, the algorithm iteratively processes each minutiae point from two images and tries to find out the number of common points on the basis of structural similarity among them. We try to find all matching pairs of minutiae between two fingerprint images with reference to a pair of chosen reference point. Once all the common minutiae points identified, the matching score can be calculated using various existing formulas.
\end{abstract}

Index Terms----Fingerprint image; template image; input image; ridge end; bifurcation; minutiae

\section{INTRODUCTION}

Biometrics is the field of science which involves recognizing humans automatically via unique characteristics. Recognizing humans by means of their fingerprint impression is the most widely used biometrics today. The fingerprint recognition started in $1600 \mathrm{~s}$, but the use of biometrics is expanding very rapidly. Fingerprints have been used widely in laptops, computers, enterprises, to college buildings etc. Fingerprint recognition is used as a means to restrict the unauthorized users from accessing useful data.

Fingerprint contains complex patterns of stripes, called ridges. There exists some gap between the ridges, called valleys. A ridge can spread further in two ways, either it ends or bifurcates into two ridges. The place where ridge ends is called termination or ridge end and where it bifurcates is called bifurcation. Minutiae consist of these two basic types, ridge end and bifurcation. The overall fingerprint recognition consists of three steps:-
a. Image Preprocessing
b. Minutiae detection and Feature extraction
c. Minutiae matching

Image preprocessing is the first step of fingerprint matching. It is done to make image clearer to understand. In this, three steps are involved, Image Quality Enhancement, Binarization and Thinning. In image quality enhancement phase the quality of the given image is improved so that the image will be good enough for further processing. The binarization of the gray scale image converts it to black and white image. A threshold is selected and applied to each pixel. The pixels above threshold are assigned black and below threshold are assigned white. In thinning, the width of each ridge is reduced to one pixel by using various methods. Central line thinning is advised.

Minutiae detection and feature extraction step involves refining of the thinned image, detecting the minutiae points and then extracting features from image. In Detection, the whole image is scanned to detect the ridge ends and bifurcations. In Extraction, the coordinates, the angle of orientation and the type of minutiae is computed for each minutiae point.

Minutiae matching, the third step involves matching the template image with the input image. Template image is collected at the time of enrollment and stored 
in the database. During recognition phase, the input image is compared against template image. This phase decides whether the two images are from the same finger or from different fingers.

We are going to propose a novel algorithm for the last step i.e. minutiae matching. The basic idea behind our algorithm is to draw all possible number of polygons by joining all detected minutiae points of a given image. This is done for both the template image and the input image. Then the polygons of one image are overlapped on other image such that maximum numbers of polygons coincide.



Fig 1: Template image

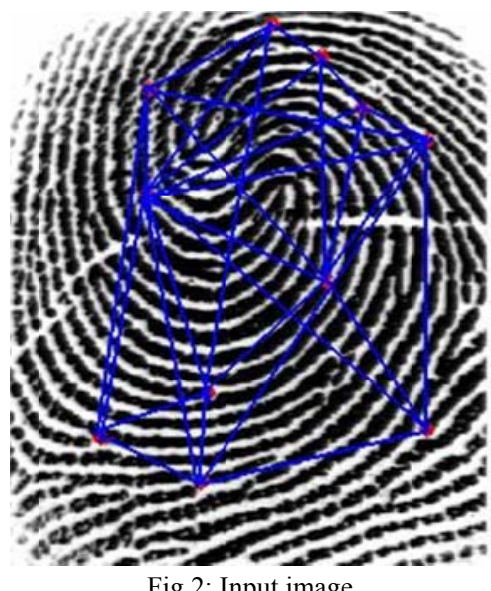

Fig 2: Input image
Two polygons can be compared for equality by simply considering the length of sides and angles made by two sides.

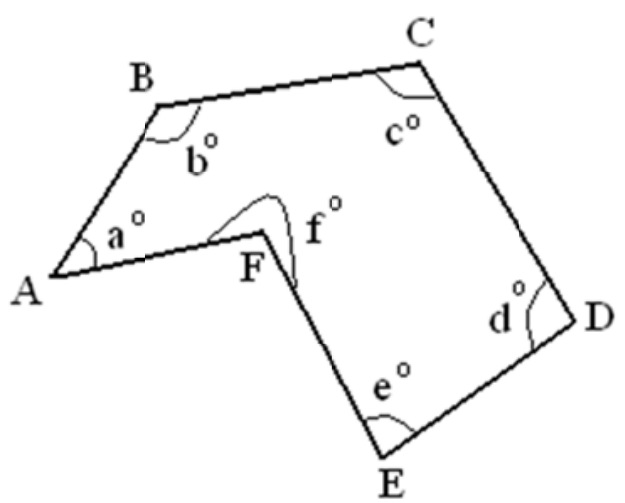

Fig 3: polygon-1



Fig 4: polygon-2

We can say polygon-1 and polgon-2 are same if the following conditions are true.

$$
\begin{aligned}
& \mathrm{AB}=\mathrm{PQ}, \mathrm{BC}=\mathrm{QR}, \mathrm{CD}=\mathrm{RS}, \mathrm{DE}=\mathrm{ST}, \mathrm{EF}=\mathrm{TU}, \\
& \mathrm{AF}=\mathrm{PU} \quad \text { and } \\
& \mathrm{a}=\mathrm{p}, \mathrm{b}=\mathrm{q}, \mathrm{c}=\mathrm{r}, \mathrm{d}=\mathrm{s}, \mathrm{e}=\mathrm{t}, \mathrm{f}=\mathrm{u}
\end{aligned}
$$

This is all about our algorithm and we match two fingerprint samples on this idea. Two fingerprints match only when they form similar polygons after joining the minutiae points. 


\section{PRIVIOUS WORK DONE ON MINUTIAE MATCHING}

A lot of work has been done successfully in this area in past. One of the most widely used methods is $[3,1]$ to create a matrix, called rotate value, of the orientation angle difference between each template minutiae, $T_{k}(1 \leq k \leq N T)$, and each input minutiae, $I_{m}$ $(1 \leq m \leq N I)$. Here, NT and NI represent the total number of minutiae in the template and input sets, respectively. The value at rotate values $(k, m)$ represents the difference between the orientation angles of $T_{k}$ and $I_{m}$.

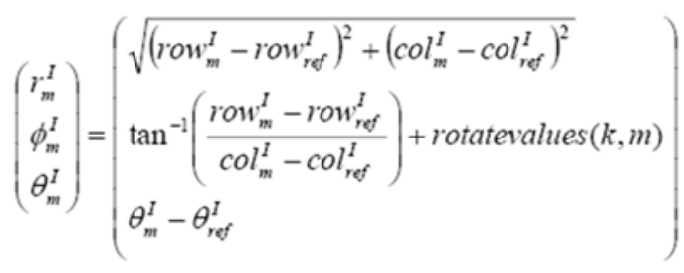

The above formula is used to calculate the radial distance, radial angle and the orientation for template image and the input image. Then these values are compared among the minutiae points, one point from template and one from input image, with certain threshold value to find the matching points.

Another method called focal point [5] considered as reference point is chosen that is designed as a center of moment in polar coordinate for the fingerprint matching method. The focal point for any type of fingerprint images can be identified and tested with various orientation, elastic distortion and noise.

Another approach for minutiae matching is done by calculating the differences of spatial and orientation of minutiae [6].

Fingerprint minutiae can be matched by using both the local and global structures of minutiae [7]. The local structure of a minutia describes a rotation and translation invariant feature of the minutia in its neighborhood. It is used to find the correspondence of two minutiae sets and increase the reliability of the global matching. The global structure of minutiae reliably determines the uniqueness of fingerprint.

According to He et al.'s [8] matching algorithm use of a variable sized bounding box makes the matching algorithm more robust to non-linear deformation between fingerprint images.

Another interesting technique was proposed [9], where the minutiae points are connected using a Delaunay triangulation and analyzes the relative position and orientation of each minutia with respect to its neighbors obtained by the triangle structure. Two fingerprints are considered matching, if their triangle structures are similar according the neighbor relationship.

Shi et al. [10] introduced two global statistical features of fingerprint image, including the mean ridge width and the normalized quality estimation of the whole image, and proposed a novel fingerprint matching algorithm based on minutiae sets combined with the global statistical features. This algorithm has advantage of both local and global features in fingerprint matching.

Xuefeng Liang and Tetsuo Asano [11] proposed a minutia polygons algorithm that is used to match distorted fingerprints. A minutia polygon describes not only the minutia type and orientation but also the minutia shape. This technique employs an improved distortion model using a Multi-quadric basis function with parameters. Adjustable parameters make this model more suitable for fingerprint distortion.

\section{PROPOSED ALGORITHM}

For our proposed algorithm we need to store the coordinates of the detected minutiae and the types of minutiae.

TABLE I. THE EXTRACTED FORMAT CAN BE SEEN AS FOLLOWS

\begin{tabular}{|c|c|}
\hline Absolute co ordinate & Minutiae Type \\
\hline 79,102 & Bifurcation \\
\hline 75,214 & Ridge End \\
\hline 64,110 & Bifurcation \\
\hline 157,182 & Ridge End \\
\hline
\end{tabular}

These details are stored for the template and the input image. From these two sets of attributes our algorithm decides whether the images are from same finger or from different finger. The detail about the algorithm is explained in the next section.

\section{ILLUSTRATION OF THE PROPOSED ALGORITHM}

Let us say the total no. of minutiae in the template is 5 and the total number of minutiae in the input image is 8 . The algorithm works as follows. 
TABLE II. FOR TEMPLATE

\begin{tabular}{|c|c|c|c|}
\hline Name & Co ordinate & $\begin{array}{c}\text { Radial } \\
\text { Distance }\end{array}$ & Minutiae Type \\
\hline A & 79,102 & 0 & Bifurcation \\
\hline B & 75,214 & 112 & Ridge end \\
\hline C & 64,110 & 17 & Bifurcation \\
\hline D & 157,182 & 111 & Ridge end \\
\hline E & 50,255 & 156 & Bifurcation \\
\hline
\end{tabular}

TABLE III. FOR INPUT IMAGE

\begin{tabular}{|c|c|c|c|}
\hline Name & Co ordinate & $\begin{array}{c}\text { Radial } \\
\text { Distance }\end{array}$ & Minutiae Type \\
\hline P & 75,103 & 0 & Bifurcation \\
\hline Q & 87,90 & 18 & Bifurcation \\
\hline R & 172,146 & 106 & Ridge end \\
\hline S & 108,206 & 108 & Ridge end \\
\hline T & 101,254 & 153 & Bifurcation \\
\hline U & 224,228 & 194 & Ridge end \\
\hline V & 187,52 & 123 & Ridge end \\
\hline W & 231,133 & 159 & Ridge end \\
\hline
\end{tabular}

The radial distance is calculated using the following formula.

$$
\sqrt{\left(\text { row }_{m}-\text { row }_{r e f}\right)^{2}+\left(\mathrm{col}_{m}-\operatorname{col}_{r e f}\right)^{2}}
$$

The radial distance is the distance of the coordinate from a chosen reference point. So if the reference point and the coordinate are the same, then the radial distance will be zero.

During pairing the minutiae we must be careful about the type of minutiae (Ridge and Bifurcation). Bifurcations should be paired with bifurcation of other image and the same is for ridges. Let us pair the reference point ' $A$ ' of template image with the reference point ' $\mathrm{P}$ ' of input image. Now other calculations are as follows.

Calculation of Angles made between minutiae points with the reference point as center. Angles can be easily calculated from the coordinates of three points.

TABLE IV. FOR TEMPLATE

\begin{tabular}{|l|l|l|l|l|}
\hline A & B & C & D & E \\
\hline B & -- & $60^{\circ}$ & $46^{\circ}$ & $9^{\circ}$ \\
\hline C & $60^{\circ}$ & -- & $106^{\circ}$ & $51^{\circ}$ \\
\hline D & $46^{\circ}$ & $106^{\circ}$ & -- & $55^{\circ}$ \\
\hline E & $9^{\circ}$ & $51^{\circ}$ & $55^{\circ}$ & -- \\
\hline
\end{tabular}

TABLE V. FOR INPUT IMAGE

\begin{tabular}{|l|l|l|l|l|l|l|l|}
\hline $\mathbf{P}$ & $\mathbf{Q}$ & $\mathbf{R}$ & $\mathbf{S}$ & $\mathbf{T}$ & $\mathbf{U}$ & $\mathbf{V}$ & $\mathbf{W}$ \\
\hline $\mathbf{Q}$ & -- & & & & & & \\
\hline $\mathbf{R}$ & $71^{\circ}$ & -- & & & & & \\
\hline $\mathbf{S}$ & $120^{\circ}$ & $48^{\circ}$ & -- & & & & \\
\hline $\mathbf{T}$ & $128^{\circ}$ & $56^{\circ}$ & $8^{\circ}$ & -- & & & \\
\hline $\mathbf{U}$ & $87^{\circ}$ & $16^{\circ}$ & $32^{\circ}$ & $40^{\circ}$ & -- & & \\
\hline $\mathbf{V}$ & $23^{\circ}$ & $48^{\circ}$ & $97^{\circ}$ & $105^{\circ}$ & $64^{\circ}$ & -- & \\
\hline $\mathbf{W}$ & $58^{\circ}$ & $13^{\circ}$ & $61^{\circ}$ & $69^{\circ}$ & $29^{\circ}$ & $35^{\circ}$ & -- \\
\hline
\end{tabular}

Here the angle $71^{\circ}$ represents the angle $\mathrm{RPQ}=\mathrm{QPR}=$ $71^{\circ}$. ' $\mathrm{P}$ ' is the chosen reference point. So the angle represents the angle made between ' $Q$ ' and ' $R$ ' made with the reference point ' $\mathrm{P}$ '.



Fig 4: Figure shows the arrangement of minutiae points on the image

The following table shows the result of matching of the minutiae points on the basis of radial distance only.

TABLE VI. RADIAL DISTANCE COMPARISON

\begin{tabular}{|c|c|c|c|c|}
\hline $\begin{array}{c}\text { Minutie } \\
\text { Points of } \\
\text { Template }\end{array}$ & $\begin{array}{c}\text { Radial } \\
\text { Distance } \\
\text { from } \\
\text { Reference } \\
\text { point }\end{array}$ & $\begin{array}{c}\text { Minutiae } \\
\text { Points of } \\
\text { Input } \\
\text { image }\end{array}$ & $\begin{array}{c}\text { Radial } \\
\text { Distance } \\
\text { from } \\
\text { Reference } \\
\text { point }\end{array}$ & $\begin{array}{c}\text { Difference } \\
\text { in radial } \\
\text { distance }\end{array}$ \\
\hline A & 0 & P & 0 & 0 \\
\hline B & 112 & S & 108 & 4 \\
\hline C & 17 & Q & 18 & 1 \\
\hline D & 111 & R & 106 & 5 \\
\hline E & 156 & T & 153 & 3 \\
\hline
\end{tabular}

If we set the threshold vialue of difference between the radial distances is 5 , then all the above points are matched. So the minutiae points paired (Only radial distance is considered) till now are as follows: 


$$
\begin{aligned}
& A-P \\
& B-S \\
& C-Q \\
& D-R \\
& E-T
\end{aligned}
$$

Now let us compare the angles made by each point with other points.

TABLE VII. ANGLE COMPARISON

\begin{tabular}{|l|l|l|l|l|}
\hline $\mathbf{A} / \mathbf{P}$ & $\mathbf{B} / \mathbf{S}$ & $\mathbf{C} / \mathbf{Q}$ & $\mathbf{D} / \mathbf{R}$ & $\mathbf{E} / \mathbf{T}$ \\
\hline $\mathbf{B} / \mathbf{S}$ & -- & & & \\
\hline $\mathbf{C} / \mathbf{Q}$ & $60^{\circ} / 120^{\circ}$ & -- & & \\
\hline $\mathbf{D} / \mathbf{R}$ & $46^{\circ} / 48^{\circ}$ & $106^{\circ} / 71^{\circ}$ & -- & \\
\hline $\mathbf{E} / \mathbf{T}$ & $9^{\circ} / 8^{\circ}$ & $51^{\circ} / 128^{\circ}$ & $55^{\circ} / 56^{\circ}$ & -- \\
\hline
\end{tabular}

Let us discuss in detail about the above table. It shows the angle $\mathrm{CAB}=60^{\circ}$ and the angle $\mathrm{QPS}=120^{\circ}$. But we have the following pairs from the previous analysis.

$$
\begin{aligned}
& A-P \\
& C-Q \\
& B-S
\end{aligned}
$$

If this is the scenario, then the angles must be same. So this contradicts and it means ' $\mathrm{C}$ ' may be a different minutiae point and not the counterpart of ' $Q$ '. The same condition is for ' $\mathrm{B}$ ' and ' $\mathrm{S}$ '. The difference between the angles is $120^{\circ}-60^{\circ}=60^{\circ}$. Let us say the threshold value for angle difference is $5^{\circ}$, and then this does not satisfy the criteria.
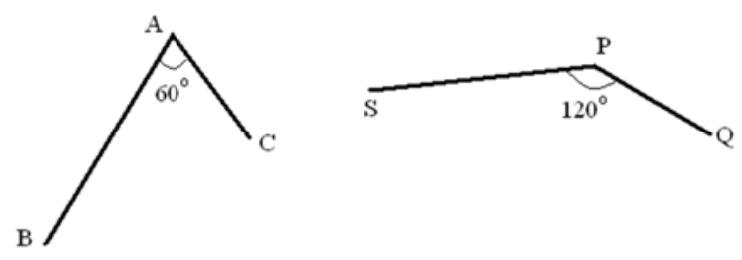

Fig 5: Figure shows the arrangement of minutiae points on the template image and the input image respectively

So if we calculate the difference between angles we find only the following angles lie below the threshold value.

Angle DAB and angle RPS, difference is $2^{\circ}$. Angle EAB and angle TPS, difference is $1^{\circ}$. Angle EAD and angle TPR, difference is $1^{\circ}$.
This implies the following pairs are correct.
A - P
$\mathrm{D}-\mathrm{R}$
$\mathrm{B}-\mathrm{S}$
$\mathrm{E}-\mathrm{T}$

So we can conclude that the given template and the input image have only 4 minutiae points matched with ' $\mathrm{A}$ ' as reference point in the template image and ' $\mathrm{P}$ ' as reference point in the input image.

The above matched minutiae points can be diagrammatically viewed as follows.

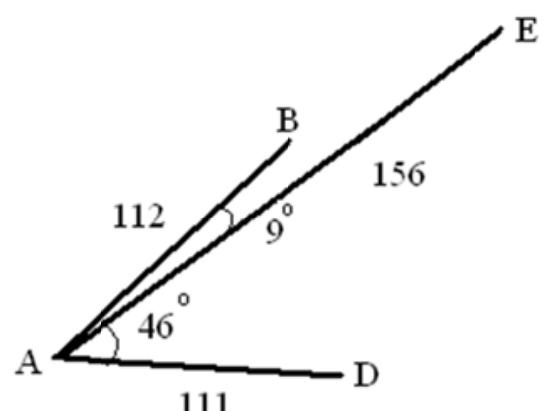

Fig 6: Figure shows the arrangement of all matched minutiae points on the templlate image

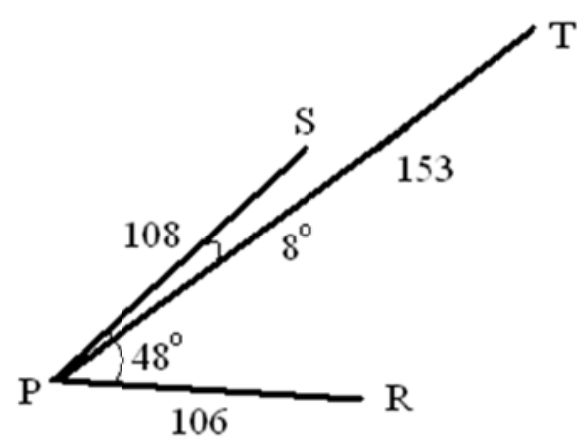

Fig 7: Figure shows the arrangement of all matched minutiae points on the input image

We can see that all the 4 minutiae points form similar structures in both the template and input image.

The same procedure is repeated for each chosen pair of reference points. The highest number of matching point is the result.

\section{THE ALGORITHM FOR MINUTIAE MATCHING}

1. Repeat step-2 to step-5 until all minutiae points consumed. 
2. Take one minutiae point from template and one from input image as reference and repeat step-3 to step-4 until all minutiae points are consumed.

3. Calculate radial distances for all points from the reference point for both the template and the input image.

4. Pair one minutiae point from template with one from input image if the difference in radial distance of the points $\leq$ certain threshold value and their type are same.

a. If the above condition is true, then find the angle between two minutiae of an image made with the reference point. Find the same angle in other image.

b. If difference between the angles $\leq$ certain threshold value, then pair minutiae points from template and input image.

5. Count the number of pairs (non repeating pairs) and this represents total number of matching minutiae points for a particular pair of reference points. Store the count value.

6. Finally select the maximum count value. This is the final matching minutiae points.

\section{CONCLUSION AND FUTURE WORK}

We have developed an algorithm for minutiae matching on the basis of structural similarity of minutiae points on the finger print image. There are only two kinds of minutiae that are considered in the above algorithm, in fact various types of minutiae can be matched using the same algorithm. We have tested the algorithm and found it efficient to a great extent. The algorithm is tad slower than some other algorithm but gives better result. In future the time complexity of the algorithm can be reduced and the same algorithm can be used in applications other than fingerprint matching where 2D structural similarity is concerned.

\section{REFERENCES}

[1] Graig T. Diefenderfer, June 2006. Thesis on "Fingerprint Recognition" at Naval Postgraduate School, Monterey, California,.

[2] Ravi J, K. B. Raja, Venugopal K. R., Fingerprint Recognition using Minutiae Score Matching, International Journal of Engineering Science and Technology Vol.1(2), 2009, 35-42.
[3] Xiping Luo, Jie Tian and Yan Wu, A Minutia Matching Algorithm in Fingerprint Verification,

[4] P. Kumar, S. R. Giri, G. R. Hegde and K. Verma, A Novel Algorithm to Extract Connected Components in a Binary Image of Vehicle License Plates, IJECCT 2012, Vol. 2 (2), Page 27-32.

[5] Krisakorn Rerkrai and Vutipong Areekul, "A New Referance Point For Fingerprint Recognition".

[6] F.A. Afsar, M. Arif and M. Hussain, Fingerprint Identification and Verification System using Minutiae matching, National Conference on Emerging Technologies 2004.

[7] Xudong Jiang and Wei-Yun Yau, Fingerprint Minutiae Matching Based on the Local And Global Structures.

[8] Yuliang He, Jie Tian, Xiping Luo, Tanghui Zhang. Image enhancement and minutiae matching in fingerprint verification. Elsevier, Pattern Recognition Letters 24 (2003) 1349-1360

[9] Giuseppe Parziale and Albert Niel. Fingerprint Matching Using Minutiae Triangulation.

[10] Peng Shi, Jie Tian, Qi Su, Xin Yang. A Novel Fingerprint Matching Algorithm Based on Minutiae and Global Statistical Features. Biometrics: Theory, Applications, and Systems, 2007. BTAS 2007. First IEEE International Conference. 27-29 Sept. 2007

[11] Xuefeng Liang and Tetsuo Asano. Fingerprint Matching Using Minutia Polygons. 18th International Conference on Pattern Recognition Volume 01.

Om Preeti Chaurasia is pursuing M.Tech in Amity University, Noida, India. She received the B.Tech degree from Noida Institute of Engineering and Technology, Noida, India in 2009. Her research areas include Data Mining, Artificial Intelligence and Software Engineering.

Saumya Ranjan Giri is a Software Engineer at IL\&FS Technologies Ltd., Bhubaneswar. He received the B.Tech degree from Biju Patnaik University of Technology, Odisha, India in 2009 and the M.Tech degree from IIIT Bhubaneswar, Odisha, India in 2011. He has published articles in International Journals and Conferences. He has presented papers in International Conferences. His current research areas include Image Processing, Data Structures and Data Compression. 
Mrs. Anchal Garg is an Assistant Professor in Amity School of Engineering and Technology, Noida, India. She received the MCA degree from Punjabi University Campus, Patiala, India in 2002 and M.Tech degree from Rajsthan Vidyapeeth, Rajasthan, India in 2006. Her research areas include Data Mining, Operations Management and Human Computer Interaction. 